\title{
Purification and Initial Characterization of a Putative Blue Light-regulated Phosphodiesterase from Escherichia coli ${ }^{\text {ๆ }}$
}

\author{
Sudarshan Rajagopal ${ }^{1}$, Jason M. Key ${ }^{1}$, Erin B. Purcell ${ }^{1}$, David J. Boerema ${ }^{1}$ and Keith Moffat ${ }^{\star, 1,2,3}$ \\ ${ }^{1}$ Department of Biochemistry and Molecular Biology, University of Chicago, Chicago, IL \\ ${ }^{2}$ Institute for Biophysical Dynamics, University of Chicago, Chicago, IL \\ ${ }^{3}$ Consortium for Advanced Radiation Sources, University of Chicago, Chicago, IL
}

Received 16 June 2004; accepted 22 August 2004

\begin{abstract}
The Escherichia coli protein YcgF contains a photosensory flavin adenine dinucleotide (FAD)-binding BLUF domain covalently linked to an EAL domain, which is predicted to have cyclic-di-guanosine monophosphate (GMP) phosphodiesterase activity. We have cloned, overexpressed and purified this protein, which we refer to as blue light-regulated phosphodiesterase (BIrp) for its putative activity. Blrp undergoes a reversible photocycle after exposure to light in which the spectrum of its photostationary state and kinetics of recovery of the dark state are similar to those of the isolated BLUF domain of the AppA protein. Unlike the AppA BLUF domain, the chromophore environment in the context of full-length Blrp is asymmetric, and the protein does not undergo any detectable global changes on exposure to blue light. When overexpressed in $E$. coli, Blrp copurifies with certain proteins which suggests that it plays a protective role in response to oxidative stress. Predicted proteins from Klebsiella pneumoniae and from a bacterium in the Sargasso Sea are similar to $E$. coli Blrp in both their BLUF and EAL domains, which suggests that blue light sensing in these bacteria may follow similar pathways.
\end{abstract}

\section{INTRODUCTION}

Light sensing is critical to organisms: it serves as a source of energy for photosynthetic organisms, functions as an important environmental stimulus and may damage cells (1). Photoreceptors sense the color of light through differences in the structure of their light-absorbing chromophores. Blue light photoreceptors include phototropins and cryptochromes, which contain flavin moieties,

\Posted on the website on 28 September 2004.

*To whom correspondence should be addressed: Department of Biochemistry and Molecular Biology, University of Chicago, 920 East 58th Street, Chicago, IL 60637, USA. Fax: 773-702-0439; e-mail: moffat@ cars.uchicago.edu

Abbreviations: ACES, N-(2-acetamido)-2-aminoethanesulfonic acid; Blrp, blue light-regulated phosphodiesterase; CD, circular dichroism; CHES, 2-(cyclohexylamino)ethanesulfonic acid; DTT, dithiothreitol; FAD, flavin adenine dinucleotide; FMN, flavin mononucleotide; GMP, guanosine monophosphate; IPTG, isopropyl-L-thio- $\beta$-galactoside; LPS, lipopolysaccharide; MS, mass spectrometry; MW, molecular weight; NTA, nitrilotriacetic acid; OD, optical density; PCR, polymerase chain reaction; TLC, thin layer chromatography; UDP, uridine diphosphate; WT, wild type.

(c) 2004 American Society for Photobiology $0031-8655 / 04 \quad \$ 5.00+0.00$ and photoactive yellow proteins, which have a $p$-coumaric acid chromophore (1). Recently, a novel flavin adenine dinucleotide (FAD)-binding blue light photoreceptor domain has been identified in cyano- and proteobacteria, the BLUF domain (2). This domain lacks significant sequence homology to other flavinbinding blue light photoreceptors (2). Five proteins that contain this domain have been isolated and shown to bind FAD: the phototactic receptors PAC $\alpha$ and PAC $\beta$ in the eukaryotic unicellular alga Euglena gracilis (3), AppA from Rhodobacter sphaeroides $(4,5)$, YcgF from Escherichia coli (4) and most recently, Slr1694 from Synechocystis (6). Proteins predicted to contain BLUF domains are linked to a number of different effector domains such as phosphodiesterases and adenylyl cyclases (2). It is therefore of interest to characterize the spectroscopic and structural properties of proteins containing a BLUF domain to identify how different domains are able to sense blue light and transduce that signal into a biological response.

PAC $\alpha$, PAC $\beta$ and AppA have been shown to act in vivo as blue light photoreceptors $(3,5,7)$; in vitro AppA and Slr1694 undergo reversible photocycles after exposure to blue light $(5,6)$ and PAC $\alpha$ and PAC $\beta$ exhibit blue light-regulated adenylyl cyclase activity (3). No such activity or photosensitivity has been demonstrated in E. coli $\mathrm{YcgF}$ in vivo or in vitro. Here, we further characterize fulllength YcgF, which contains a BLUF domain covalently linked to an EAL domain, predicted to have cyclic-di-guanosine monophosphate (GMP) phosphodiesterase activity (8). Therefore, we refer to YcgF as blue light-regulated phosphodiesterase (Blrp). Unlike $R$. sphaeroides and E. gracilis, E. coli does not have many well-defined, blue light-sensitive responses; one of the few such responses is a photophobic response to intense levels of blue light (9-13). Therefore, it is also of interest to explore the biological role that Blrp plays because it may allow new light-regulated pathways in $E$. coli to be identified.

\section{MATERIALS AND METHODS}

Cloning and expression of Blrp. A genomic midiprep of E. coli strain JM101 was performed, followed by polymerase chain reaction $(\mathrm{PCR})$ with $\mathrm{N}$ terminal (5'-TATAGAATTCATGCTTACCACCCTTATTTATCG-3') and C-terminal (5'-TATACTCGAGTTATTTTTTCTCTGGCCACGCT- $3^{\prime}$ ) primers to amplify the segment encoding Blrp, flanked by EcoRI and XhoI restriction sites. The resultant PCR product was restriction digested and ligated into the pET-28a $(+)$ expression vector, encoding an N-terminal hexahistidine tag and kanamycin resistance (EMD Biosciences, La Jolla, CA). This construct was transformed into BL21(DE3)-RIL cells, which were grown to an optical density (OD) of $0.5-0.7$ at $37^{\circ} \mathrm{C}$ followed by induction with $100 \mu M$ isopropyl-L-thio- $\beta$-galactoside (IPTG) and overnight growth at 
$20^{\circ} \mathrm{C}$. We found that the protein derived from this construct, although it expressed well and was quite soluble, precipitated within a few days after purification. Therefore, we also generated a construct with a C-terminal Histag using the same vector. An NcoI cut site was generated in the N-terminal primer by mutation of the N-terminal MLT residues to MVT (5'TATACCATGGTGCTTACCACCCTTATTTATCG-3'), and a HindIII site was generated in the C-terminal primer ( $5^{\prime}$-GGTATCGCACCGGTCTCTTTTTCGAATATA-3'). The resulting fragment encoding Blrp was ligated into $\mathrm{pET}-28 \mathrm{~b}(+)$ to generate a construct with a C-terminal hexahistidine tag. After expression and purification using similar conditions, the protein derived from this construct was considerably more stable; all experiments were performed on this protein unless otherwise stated.

Expression and purification of Blrp. Escherichia coli BL21 DE3 cells were transformed with the C-terminal hexahistidine construct described above and grown to an $\mathrm{OD}$ of 0.5 at $37^{\circ} \mathrm{C}$, cooled to $20^{\circ} \mathrm{C}$ and then induced with $100 \mu M$ IPTG and grown overnight. Cells, which had a distinct yellow color, were spun down, resuspended in $50 \mathrm{~m} M$ Tris, $\mathrm{pH} 8.0,300 \mathrm{mM} \mathrm{NaCl}$, $10 \mathrm{~m} M$ imidazole and $1 \mathrm{~m} M$ dithiothreitol (DTT) (resuspension buffer) with $200 \mu M$ phenylmethane sulfonyl fluoride. The cells were lysed by incubation with lysozyme for $30 \mathrm{~min}$ followed by sonication. After centrifugation at $20000 \mathrm{rpm}$ for $30 \mathrm{~min}$, the supernatant was applied to a Ni-NTA agarose column (Qiagen, Valencia, CA), washed with the resuspension buffer and eluted with the resuspension buffer $+250 \mathrm{~m} M$ imidazole. Protein was then applied to a gel filtration column in $25 \mathrm{~m} M$ Tris, $50 \mathrm{mM} \mathrm{NaCl}, 25 \mathrm{mM} \mathrm{KCl}$, $25 \mathrm{~m} M \mathrm{MgCl}_{2}, 1 \mathrm{~m} M$ DTT and $\mathrm{pH}$ 8.0; unless otherwise stated, all subsequent measurements were made under these solution conditions. A gel filtration column packed with S200HR media (Sigma, St. Louis, MO) was run at a flow rate of $1 \mathrm{~mL} \mathrm{~min}^{-1}$ using an AKTAprime FPLC system (Amersham Biosciences, Piscataway, NJ) and calibrated with high and low molecular weight (MW) standards (Amersham Biosciences). The dead volume of the column was approximately $48 \mathrm{~mL}$. To maintain intact protein samples over weeks to months, HALT ${ }^{\mathrm{TM}}$ protease inhibitor cocktail (Pierce, Rockford, IL) was added. Protein concentration was estimated using the Bradford assay (Bio-Rad, Hercules, CA) with bovine gamma globulin as a standard; these results were consistent with the estimate of protein concentration using an extinction coefficient at $462 \mathrm{~nm}$ for protein-bound FAD of $11.3 \mathrm{~m} M^{-1} \mathrm{~cm}^{-1}$ (14).

Absorption spectroscopy. Measurements using Cary 500 UV scanning and HP 8453 diode-array spectrophotometers were made with a protein sample diluted to an $\mathrm{OD}_{452}$ of $\sim 0.7$ at $25^{\circ} \mathrm{C}$. Broadband blue light (400-500 $\mathrm{nm}$ ) from a mercury lamp of $100 \mu \mathrm{mol} \mathrm{m} \mathrm{m}^{-2} \mathrm{~s}^{-1}$ intensity was used for excitation and generation of a photostationary state. Fits to exponential decays were obtained in Origin 7.0 (OriginLab, Northampton, MA). Singular value decomposition was performed based on a subroutine by Press (15). To test the $\mathrm{pH}$ dependence of the decay back to the dark state, samples at $\mathrm{pH} 6$ were buffered in $25 \mathrm{mM}$ 2-( $N$-morpholino)-ethanesulphonic acid, samples at $\mathrm{pH} 7$ in $25 \mathrm{~m} M$ ACES and samples at $\mathrm{pH} 8-10$ in $25 \mathrm{~m} M$ CHES with all other buffer conditions (salt and $1 \mathrm{~m} M$ DTT) kept the same as above.

Visible circular dichroism spectroscopy. Visible circular dichroism (CD) measurements at $25^{\circ} \mathrm{C}$ were made from 550 to $300 \mathrm{~nm}$ using an AVIV Circular Dichroism Spectrometer Model 202, at a protein concentration of $60 \mu M$. Spectra of the photostationary state were obtained by illumination with white light from a halogen lamp for $30 \mathrm{~s}$ before collection of the spectrum. Because the scan rate was $15 \mathrm{~nm} \mathrm{~min}^{-1}$, a scan took $16.67 \mathrm{~min}$; thus the extent of photoactivation of the last measurement at $300 \mathrm{~nm}$ had decayed to $\sim 20 \%$ to that of the first measurement taken at $550 \mathrm{~nm}$. The light-dark difference CD spectrum was corrected to account for this difference in photoactivation. Light and dark absorption spectra were calculated from the dynode voltage to confirm that the protein was undergoing a photocycle.

Thin layer chromatography. Sample preparation for thin layer chromatography (TLC) was performed as given by Christie et al. (16). After NiNTA purification, the protein sample was boiled in $70 \%$ ethanol for 2 min to release the chromophore. After centrifugation, the supernatant was applied to a Baker-flex silica gel IB2-F strip (J.T. Baker, Inc., Phillipsburg, NJ) with a mobile phase of $n$-butanol-acetic acid-water (3:1:1 by volume) (17). Saturated solutions of FAD, FMN and riboflavin (Sigma) in $70 \%$ ethanol were run as standards.

Sedimentation velocity analysis. Sedimentation velocity analysis was performed using a Beckman Optima XL-A analytical ultracentrifuge with an AN-60 rotor. Samples of $\mathrm{OD}_{452} \sim 0.5$ in the $1.2 \mathrm{~mm}$ path length, twochannel aluminum centerpiece were centrifuged at $35000 \mathrm{rpm}$ for $15 \mathrm{~h}$ at $22^{\circ} \mathrm{C}$ while monitoring the absorbance at $452 \mathrm{~nm}$. The data was analyzed in Ultrascan 6.2 (18), and after removal of those scans that had yet to clear the meniscus or did not show a plateau, van Holde-Weischet analysis was performed to calculate the sedimentation coefficient (18).

Mass spectrometry fingerprinting. Ni-NTA-purified protein was run on an sodium dodecyl sulfate-polyacrylamide gel electrophoresis gel, and those bands that reproducibly copurified with Blrp were excised and subjected to trypsin digestion followed by mass spectrometry (MS) fingerprinting.

\section{RESULTS}

\section{Cloning, expression and purification of Blrp}

A full-length construct of Blrp with a C-terminal hexahistidine affinity tag expressed well in E. coli BL21(DE3) cells and was then purified by Ni affinity and gel filtration chromatography. After this purification, the protein still bound its flavin tightly and was stable for several weeks at $4^{\circ} \mathrm{C}$. As seen in Fig. 1A, Blrp consistently copurifies with a small number of high and low MW components after affinity chromatography (see below); two high MW components were originally observed to consistently copurify with Blrp in its original isolation by Gomelsky and Kaplan (4). The absorption spectrum of Blrp (Fig. 1B) is characteristic of a flavoprotein and is similar to the previously reported spectrum (4), with an absorption maximum at $458 \mathrm{~nm}$. TLC analysis of the chromophore $\left(\mathrm{R}_{\mathrm{f}}=0.19\right)$ was consistent with it being FAD $\left(\mathrm{R}_{\mathrm{f}}=0.17\right)$ and not FMN $\left(\mathrm{R}_{\mathrm{f}}=\right.$ $0.31)$ or riboflavin $\left(\mathrm{R}_{\mathrm{f}}=0.59\right)$.

\section{Photocycle}

The spectra of the dark state and of the light, photostationary state obtained after illumination of the dark state form with broadband blue light for $30 \mathrm{~s}$ are shown in Fig. 2A. The dark spectrum has a peak at $458 \mathrm{~nm} ; 13 \mathrm{~nm}$ redshifted relative to that of AppA (5). As in AppA $(5,19)$, the light spectrum is redshifted relative to the dark state spectrum by about $4 \mathrm{~nm}$, resulting in an absorption maximum at $462 \mathrm{~nm}$. The light-dark difference spectrum is shown in Fig. 2B; this spectrum is qualitatively similar to that of AppA in form with maxima at 398, 470 and $506 \mathrm{~nm}$ and minima at 336, 382, 428, 452 and $483 \mathrm{~nm}$. The fluence response curve of Blrp based on $\left(\Delta \mathrm{A}_{506}-\right.$ $\Delta \mathrm{A}_{452}$ ) is shown in Fig. $2 \mathrm{C}$.

The kinetics of recovery from the photostationary state to the dark state monitored at 452 and $506 \mathrm{~nm}$ each can be fit by single exponentials with relaxation times close to $10 \mathrm{~min}$ (data not shown), corresponding to a half-life of $6.93 \mathrm{~min}$, which is approximately two times shorter than the reported half-life of AppA $(5,19)$. However, both traces exhibit an additional, slower relaxation from the light state to the dark state that are visible in a $\log \left(\Delta \mathrm{A}(\mathrm{t}) / \Delta \mathrm{A}\left(\mathrm{t}_{0}\right)\right)$ $v s$ time plot (Fig. 3A). Singular value decomposition of the timedependent difference spectra in the wavelength range of 300-550 $\mathrm{nm}$ and time range from 0 to 50 min was performed to identify the number of states present during the decay. This analysis shows that there are two species present (Fig. 3B): the first constitutes $85 \%$ of the data and is well described by a single decay of $\sim 10.7$ min (with a possible slight contamination by the slower decaying species); the second constitutes $15 \%$ of the data, the majority of which evolves at a rate so low that we were unable to estimate it from these data (Fig. 3B,C). The two species-associated difference spectra are shown in Fig. 3D. The first species has a difference spectrum similar to that in Fig. 2B and the light-minus-dark spectra of AppA $(5,19)$; the second species has a qualitatively different spectrum. The second species may be due to a small amount of irreversible aggregation, as evidenced by the upward trajectory of its time course at long times, which is further enhanced on exposure to light. In this regard, 

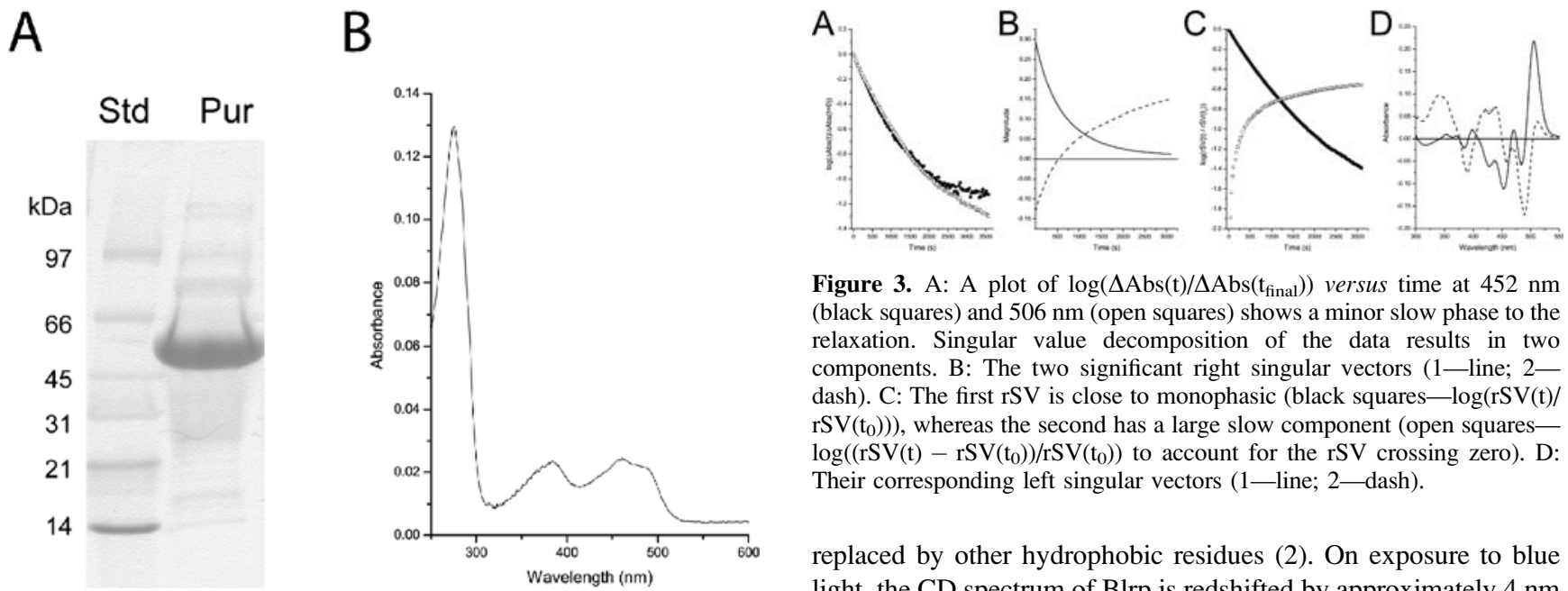

Figure 3. A: A plot of $\log \left(\Delta \mathrm{Abs}(\mathrm{t}) / \Delta \mathrm{Abs}\left(\mathrm{t}_{\text {final }}\right)\right)$ versus time at $452 \mathrm{~nm}$ (black squares) and $506 \mathrm{~nm}$ (open squares) shows a minor slow phase to the relaxation. Singular value decomposition of the data results in two components. B: The two significant right singular vectors (1-line; 2dash). C: The first rSV is close to monophasic (black squares- $\log (\mathrm{rSV}(\mathrm{t}) /$ $\left.\mathrm{rSV}\left(\mathrm{t}_{0}\right)\right)$ ), whereas the second has a large slow component (open squares$\log \left(\left(\mathrm{rSV}(\mathrm{t})-\mathrm{rSV}\left(\mathrm{t}_{0}\right)\right) / \mathrm{rSV}\left(\mathrm{t}_{0}\right)\right)$ to account for the rSV crossing zero). D: Their corresponding left singular vectors (1-line; 2 -dash).

replaced by other hydrophobic residues (2). On exposure to blue light, the CD spectrum of Blrp is redshifted by approximately $4 \mathrm{~nm}$ (Fig. 4A), resulting in the light-dark visible CD difference spectrum shown in Fig. 4B. This spectrum is not similar to the light-dark absorption difference spectrum in Fig. 2B, although a peak at 506 $\mathrm{nm}$ is common to the two.

although the primary component of the difference spectrum is reversible, the photocycle cannot be considered as fully reversible.

The rate of dark state recovery increases with higher $\mathrm{pH}$ (Table 1), as also observed for AppA (19). Measurements were restricted from the $\mathrm{pH}$ range from 6.4 to 10 : above $\mathrm{pH} 10$ the protein precipitated quickly in the dark state, whereas below $\mathrm{pH} 6.4$, the protein precipitated slightly, but on exposure to light, precipitated quickly (data not shown). Evidently, there are differences in $\mathrm{pH}$ stability between the dark and light states.

\section{Chromophore environment}

Visible CD spectroscopy reports on the local environment of the flavin chromophore. These measurements from 300 to $550 \mathrm{~nm}$ on dark and light state Blrp are shown in Fig. 4A. Unlike WT AppA whose visible $C D$ spectrum is positive throughout this range (20), that of Blrp is positive from 300 to $375 \mathrm{~nm}$ and negative from 375 to $525 \mathrm{~nm}$. This spectrum suggests that there is asymmetry in the chromophore environment between the isoalloxazine ring and either the ribityl side chain or the surrounding protein environment (20-22). A similar visible CD spectrum with positive and negative components has been observed in the Y21F AppA mutant (20), which is unable to photocycle. It is unlikely that the asymmetry of the FAD environment in Blrp is due to the substitution of residue 2, leucine in wild type, by valine in our construct. Although this position is leucine in the majority of BLUF domains, it can be
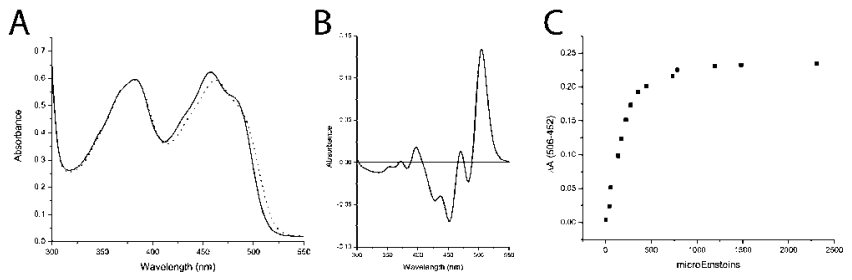

Figure 2. Absorption spectra and recovery kinetics of the Blrp photostationary state. A: Absorption spectra for dark-adapted (line) and on continuous illumination with blue light, light (dot) states. B: Light-dark difference spectrum. C: Fluence response curve.

\section{Oligomeric state}

The retention time of Blrp in gel filtration (Fig. 5A) corresponds to a MW of $48 \mathrm{kDa}$ (Fig. 5B), close to the expected monomer MW of $47 \mathrm{kDa}$. The shoulder at earlier retention times of this peak is likely due to the high MW components from Fig. 1A because it does not exhibit the Blrp absorption spectrum. On exposure to light, there is no significant change in its elution profile (data not shown). Sedimentation velocity analysis of Blrp after gel filtration is consistent with the gel filtration of the dark state, yielding a sedimentation coefficient of $\sim 4.95 \mathrm{~S}$ with little sample heterogeneity (data not shown), close to the $4.87 \mathrm{~S}$ coefficient predicted for a sphere of $47 \mathrm{kDa} \mathrm{MW}$. Taken alone, this result does not conclusively establish that the protein is a monomer. A similar sedimentation coefficient of 5.01 S could arise from a dimer arranged as a prolate ellipsoid; however, this would not be consistent with the results from gel filtration chromatography. We conclude that Blrp is a monomer in solution and that the darkadapted protein does not undergo a structural change detectable by gel filtration on exposure to blue light.

\section{Possible biological signaling partners}

We were unable to assess Blrp activity because cyclic-di-GMP is not commercially available (although it can be synthesized [23]). We sought but did not observe activity toward cyclic adenosine monophosphate or cyclic GMP (data not shown), although our assay would not be able to detect the low levels of activity associated with the EAL domain of the $E$. coli protein Dos $(\sim 0.15$ $\min ^{-1}$ ) (24). These levels are so low that they are unlikely to be

Table 1. Recovery rate of dark state from a photostationary state at different $\mathrm{pH}$

\begin{tabular}{rc}
\hline $\mathrm{pH}$ & Rate $\left(\mathrm{s}^{-1}\right)$ \\
\hline 7.0 & $1.70 \times 10^{-3}$ \\
8.0 & $2.15 \times 10^{-3}$ \\
9.0 & $2.81 \times 10^{-3}$ \\
10.0 & $4.69 \times 10^{-3}$ \\
\hline
\end{tabular}



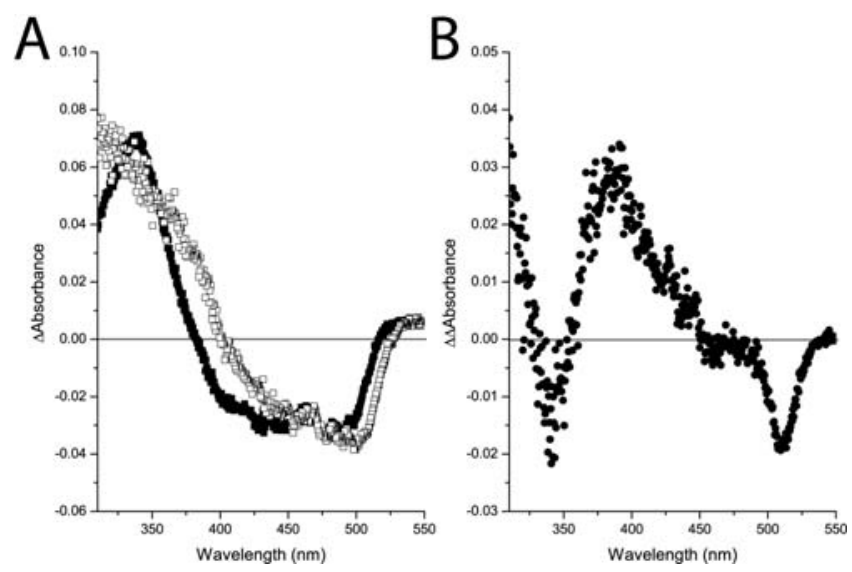

Figure 4. Asymmetric flavin environment in full-length Blrp. A: Visible $\mathrm{CD}$ of Blrp in dark (closed squares) and light (open squares) states. B: Light-dark difference CD spectrum.

physiological. To elucidate the biological role of Blrp, we sought to identify those proteins that repeatedly copurified with it on overexpression in E. coli. As mentioned earlier, Gomelsky and Kaplan originally observed two high MW major proteins that copurified with Blrp (4). In our study, some proteins that copurified with Blrp, such as ferric uptake regulator (histidine rich), GroEL (chaperone), DnaK (chaperone) and peptidyl prolyl cis-trans isomerase (histidine rich), were not regarded as significant because they were likely to be contaminants (they either contained stretches of histidines that promote retention on the $\mathrm{Ni}$ affinity column or were chaperones). The two proteins that consistently copurified with Blrp and are unlikely to be contaminants are two high MW proteins: glucosamine-6-phosphate synthase and uridine diphosphate (UDP)-D-glucuronate dehydrogenase, which are both involved in pathways regulating the decoration of the membrane with sugars. Their presence may be consistent with Blrp serving a photoprotective function in E. coli (see below).

\section{DISCUSSION}

\section{Similarities and differences to AppA}

We have isolated and spectroscopically characterized a putative blue light-responsive phosphodiesterase from E. coli. On exposure to blue light, it exhibits a photocycle similar to that of AppA in kinetics and difference spectrum. However, Blrp displays significant differences from the BLUF domain of AppA: its visible CD spectrum shows asymmetry of the FAD environment, unlike WT AppA, and it does not display the global structural changes on exposure to light that were characteristic of AppA (5). These differences may arise from the context of the BLUF domain in the full-length Blrp as opposed to an isolated, single domain in AppA. That is, the presence of the EAL domain may affect the chromophore-binding BLUF domain. Structural changes in the BLUF domain associated with formation of the light state must also be coupled in some manner to a signaling partner or effector domain. In the isolated BLUF domain of AppA alone this change is present, but in the context of the full-length Blrp protein that contains an intramolecular signaling partner, it is not observed by gel filtration.
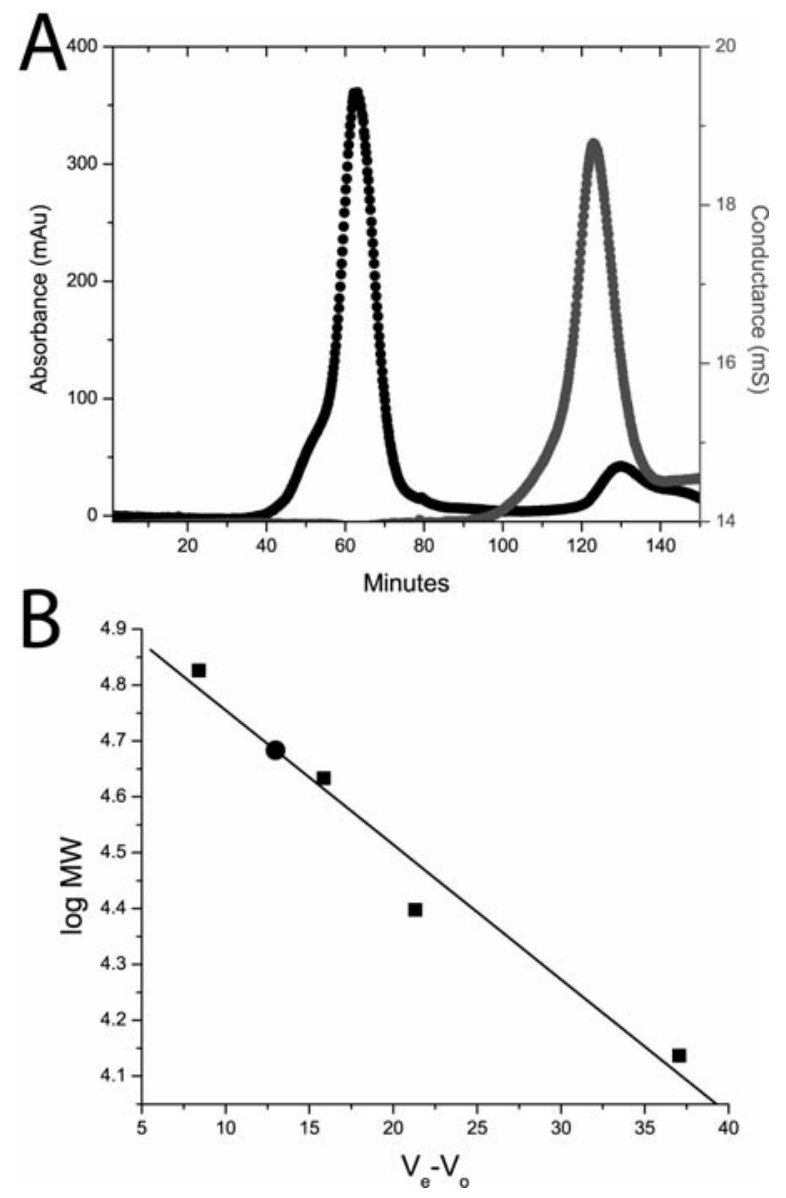

Figure 5. Gel filtration chromatography of Blrp in the dark state. A: Elution profile of dark state Blrp (black), which is nearly identical to that for light state Blrp (not shown). A conductivity trace showing free flavin is in gray. B: Calibration of the column with molecular weight standards (squares), showing Blrp (circle) elutes with a predicted molecular weight of $48 \mathrm{kDa}$.

\section{A possible biological role for Blrp}

A well-documented blue light response in E. coli is a photophobic response to levels of blue light sufficiently intense that they are unlikely to be physiological (9-12). Indeed, this response may arise from sensitivity to reactive oxygen species generated by porphyrins in the presence of light and not from direct absorption of blue light. Mutants in the heme biosynthetic pathway show defects in the photophobic response, presumably due to the buildup of free porphyrins that generate reactive species when irradiated with blue light (13). The proteins that copurify with Blrp are involved in the attachment of 4-amino-4-deoxy-L-arabinose (LAra4N) to lipopolysaccharide (LPS) in the plasma membrane: glucosamine 6-phosphate synthase (25), which is an important regulator of the biosynthesis of amino sugar-containing macromolecules such as LPS, and the transformylase ArnA (26), which converts UDP-glucose to UDP-L-Ara4N. Light-induced damage in the presence of the photosensitizer TMPyP, which is similar to the oxidative damage caused by porphyrins on exposure to blue light, causes significant damage to the plasma membrane as well as to the protein and genetic material in the bacteria $(27,28)$. It is possible that a photoprotective function may be associated with the decoration of the plasma membrane with L-Ara4N, which could be controlled by Blrp on exposure to blue light. 


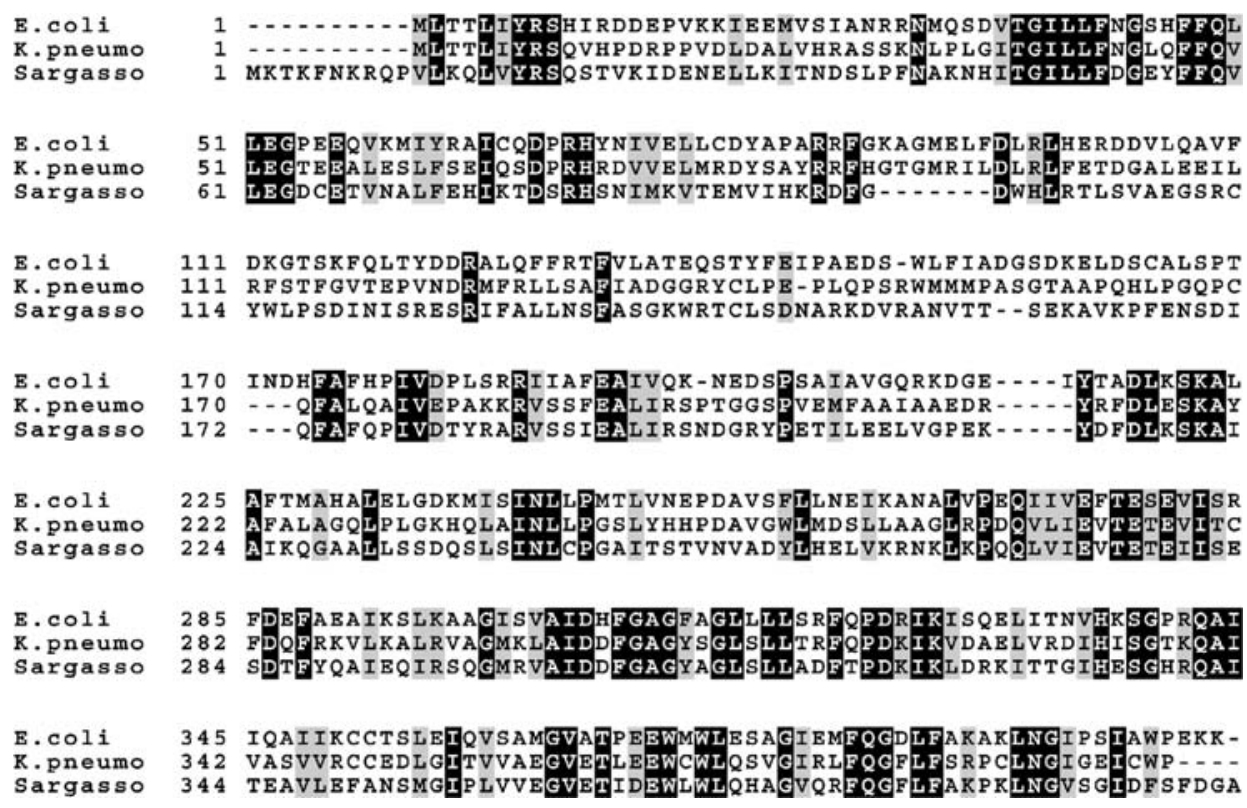

Figure 6. Alignment of the protein sequences of Blrp from Escherichia coli, Klebsiella pneumoniae and an unknown bacterium from the Sargasso Sea. Identical residues between all three proteins are boxed in black and similar residues between all three are boxed in gray.

\section{BIrp in other bacteria}

High homology to both the BLUF and EAL domains of Blrp is observed in predicted proteins from Klebsiella pneumoniae $(40 \%$ identity and 58\% similarity) (2) and a bacterium that was shotgun sequenced in the Sargasso Sea (33\% identity and 52\% similarity), which was most likely to be a member of the proteobacteriaciae (29) (Fig. 6). It is possible that these proteins play similar roles in environmental sensing in these bacteria, and possibly even play a role in response to oxidative stress caused by high light conditions. Although Blrp are present in these bacteria, they are absent in other sequenced genomes from the proteobacteriaciae, suggesting that the cellular pathway associated with this protein may be limited in distribution.

Acknowledgements - We thank Mohammed Yousef at the University of Chicago Biophysical Core facility for help with $\mathrm{CD}$ and sedimentation velocity data acquisition and interpretation, Dick Winant at the Stanford PAN Sequencing facility and Alex Schilling at the University of Chicago proteomics facility. We also thank Klaas Hellingwerf, Wouter Hoff and Sean Crosson for valuable discussions and Klaas Hellingwerf for a critical reading of this manuscript. D.B. thanks Ron Beavis for liquid chromatography-MS-MS suggestions. Supported by NIH grant GM36452 to K.M.

\section{REFERENCES}

1. Hellingwerf, K. J. (2002) The molecular basis of sensing and responding to light in microorganisms. Antonie Leeuwenhoek 81, 51-59.

2. Gomelsky, M. and G. Klug (2002) BLUF: a novel FAD-binding domain involved in sensory transduction in microorganisms. Trends Biochem. Sci. 27, 497.

3. Iseki, M., S. Matsunaga, A. Murakami, K. Ohno, K. Shiga, K. Yoshida, M. Sugai, T. Takahashi, T. Hori and M. Watanabe (2002) A blue-lightactivated adenylyl cyclase mediates photoavoidance in Euglena gracilis. Nature 415, 1047-1051.

4. Gomelsky, M. and S. Kaplan (1998) AppA, a redox regulator of photosystem formation in Rhodobacter sphaeroides 2. 4.1, is a flavoprotein. Identification of a novel fad binding domain. J. Biol. Chem. 273, 35319-35325.
5. Masuda, S. and C. Bauer (2002) AppA is a blue light photoreceptor that antirepresses photosynthesis gene expression in rhodobacter sphaeroides. Cell 110, 613 .

6. Masuda, S., K. Hasegawa, A. Ishii and T. A. Ono (2004) Light-induced structural changes in a putative blue-light receptor with a novel FAD binding fold sensor of blue-light using FAD (BLUF); Slr1694 of synechocystis sp. PCC6803. Biochemistry 43, 5304-5313.

7. Braatsch, S., M. Gomelsky, S. Kuphal and G. Klug (2002) A single flavoprotein, AppA, integrates both redox and light signals in Rhodobacter sphaeroides. Mol. Microbiol. 45, 827-836.

8. Galperin, M. Y., A. N. Nikolskaya and E. V. Koonin (2001) Novel domains of the prokaryotic two-component signal transduction systems. FEMS Microbiol. Lett. 203, 11-21.

9. Macnab, R. and D. E. Koshland Jr. (1974) Bacterial motility and chemotaxis: light-induced tumbling response and visualization of individual flagella. J. Mol. Biol. 85, 399-406.

10. Taylor, B. L. and D. E. Koshland Jr. (1975) Intrinsic and extrinsic light responses of Salmonella typhimurium and Escherichia coli. J. Bacteriol. 123, 557-569.

11. Taylor, B. L., J. B. Miller, H. M. Warrick and D. E. Koshland Jr. (1979) Electron acceptor taxis and blue light effect on bacterial chemotaxis. J. Bacteriol. 140, 567-573.

12. Yang, H., H. Inokuchi and J. Adler (1995) Phototaxis away from blue light by an Escherichia coli mutant accumulating protoporphyrin IX. Proc. Natl. Acad. Sci. USA 92, 7332-7336.

13. Yang, H., A. Sasarman, H. Inokuchi and J. Adler (1996) Non-iron porphyrins cause tumbling to blue light by an Escherichia coli mutant defective in hemG. Proc. Natl. Acad. Sci. USA 93, 2459-2463.

14. Whitby, L. G. (1953) A new method for preparing flavin-adenine dinucleotide. Biochem. J. 54, 437-442.

15. Press, W. H. (1996) Numerical Recipes in Fortran 77: The Art of Scientific Computing, 2nd ed. Cambridge University Press, Cambridge UK.

16. Christie, J. M., M. Salomon, K. Nozue, M. Wada and W. R. Briggs (1999) LOV (light, oxygen, or voltage) domains of the blue-light photoreceptor phototropin (nph1): binding sites for the chromophore flavin mononucleotide. Proc. Natl. Acad. Sci. USA 96, 8779-8783.

17. Raibekas, A. A. (1991) Green flavoprotein from P. leiognathi: purification, characterization and identification as the product of the lux G(N) gene. J. Biolumin. Chemilumin. 6, 169-176.

18. Demeler, B., J. Behlke and O. Ristau (2000) Molecular parameters from sedimentation velocity experiments: whole boundary fitting using 
approximate and numerical solutions of Lamm equation. Methods Enzymol. 321, 38-66.

19. Laan, W., M. A. van der Horst, I. H. van Stokkum and K. J. Hellingwerf (2003) Initial characterization of the primary photochemistry of AppA, a blue-light-using flavin adenine dinucleotide-domain containing transcriptional antirepressor protein from Rhodobacter sphaeroides: a key role for reversible intramolecular proton transfer from the flavin adenine dinucleotide chromophore to a conserved tyrosine? Photochem. Photobiol. 78, 290-297.

20. Kraft, B. J., S. Masuda, J. Kikuchi, V. Dragnea, G. Tollin, J. M. Zaleski and C. E. Bauer (2003) Spectroscopic and mutational analysis of the bluelight photoreceptor AppA: a novel photocycle involving flavin stacking with an aromatic amino acid. Biochemistry 42, 6726-6734.

21. Edmondson, D. E. and G. Tollin (1971) Circular dichroism studies of the flavin chromophore and of the relation between redox properties and flavin environment in oxidases and dehydrogenases. Biochemistry 10, 113-124.

22. Macheroux, P., S. Hill, S. Austin, T. Eydmann, T. Jones, S. O. Kim, R. Poole and R. Dixon (1998) Electron donation to the flavoprotein NifL, a redox-sensing transcriptional regulator. Biochem. J. 332, 413-419.

23. Hayakawa, Y., R. Nagata, A. Hirata, M. Hyodo and R. Kawai (2003) A facile synthesis of cyclic bis $\left(3^{\prime}->5^{\prime}\right)$ diguanylic acid. Tetrahedron $\mathbf{5 9}$, 6465-6471.
24. Sasakura, Y., S. Hirata, S. Sugiyama, S. Suzuki, S. Taguchi, M. Watanabe, T. Matsui, I. Sagami and T. Shimizu (2002) Characterization of a direct oxygen sensor heme protein from Escherichia coli. Effects of the heme redox states and mutations at the heme-binding site on catalysis and structure. J. Biol. Chem. 277, 23821-23827.

25. Milewski, S. (2002) Glucosamine-6-phosphate synthase-the multifacets enzyme. Biochim. Biophys. Acta 1597, 173-192.

26. Breazeale, S. D., A. A. Ribeiro and C. R. Raetz (2002) Oxidative decarboxylation of UDP-glucuronic acid in extracts of polymyxinresistant Escherichia coli. Origin of lipid a species modified with 4amino-4-deoxy-L-arabinose. J. Biol. Chem. 277, 2886-2896.

27. Nitzan, Y. and H. Ashkenazi (2001) Photoinactivation of Acinetobacter baumannii and Escherichia coli B by a cationic hydrophilic porphyrin at various light wavelengths. Curr. Microbiol. 42, 408-414.

28. Salmon-Divon, M., Y. Nitzan and Z. Malik (2004) Mechanistic aspects of Escherichia coli photodynamic inactivation by cationic tetra-meso(Nmethylpyridyl)porphine. Photochem. Photobiol. Sci. 3, 423-429.

29. Venter, J. C., K. Remington, J. F. Heidelberg, A. L. Halpern, D. Rusch, J. A. Eisen, D. Wu, I. Paulsen, K. E. Nelson W. Nelson, D. E. Fouts, S. Levy, A. H. Knaf, M. W. Lomas, K. Nealson, O. White, J. Peterson, J. Hoffman, R. Parsons, H. Baden-Tillson, C. Pfann Koch, Y.-H. Rogers and H. O. Smith (2004) Environmental genome shotgun sequencing of the Sargasso Sea. Science 304, 66-74. 